\title{
Measurement of atmospheric transparency in Telescope Array using Central Laser Facility
}

\author{
T. Uehama ${ }^{* a}$, T. Tomida $^{a}$, K. Yamazaki $^{b}$, S. UDO ${ }^{b}$, and the Telescope Array \\ Collaboration \\ ${ }^{a}$ Shinshu University, Wakasato, Nagano, Japan \\ ${ }^{b}$ Kanagawa University, Yokohama, Kanagawa, Japan \\ E-mail: 18w2026beshinshu-u.ac.jp (T.Uehama)
}

\begin{abstract}
The Telescope Array (TA) experiment has three fluorescence detector(FD) stations to detect fluorescence emission by extensive air showers induced by Ultra High Energy Cosmic Rays(UHECRs). These fluorescence light is attenuated and lost by aerosols in the atmosphere through the path of propagation to the FD. Calibration of atmospheric attenuation is necessary to estimate the energy of cosmic ray. The Central Laser Facility (CLF) consists of a laser that fires a $355 \mathrm{~nm}$ burst vertically with $10 \mathrm{~Hz}$, every half-hour. The Vertical Aerosol Optical Depth(VAOD) which is atmospheric transparency can be measured every 30 minutes by the CLF. Therefore, we can monitor the change of the atmosphere every 30 minutes within the FD observation time. The CLF is located at the center of three FD stations and observes the atmospheric transparency of the TA site. We report the results of atmospheric transparency at the TA site.
\end{abstract}

36th International Cosmic Ray Conference -ICRC2019-

July 24th - August 1st, 2019

Madison, WI, U.S.A.

\footnotetext{
${ }^{*}$ Speaker.
} 


\section{Introduction}

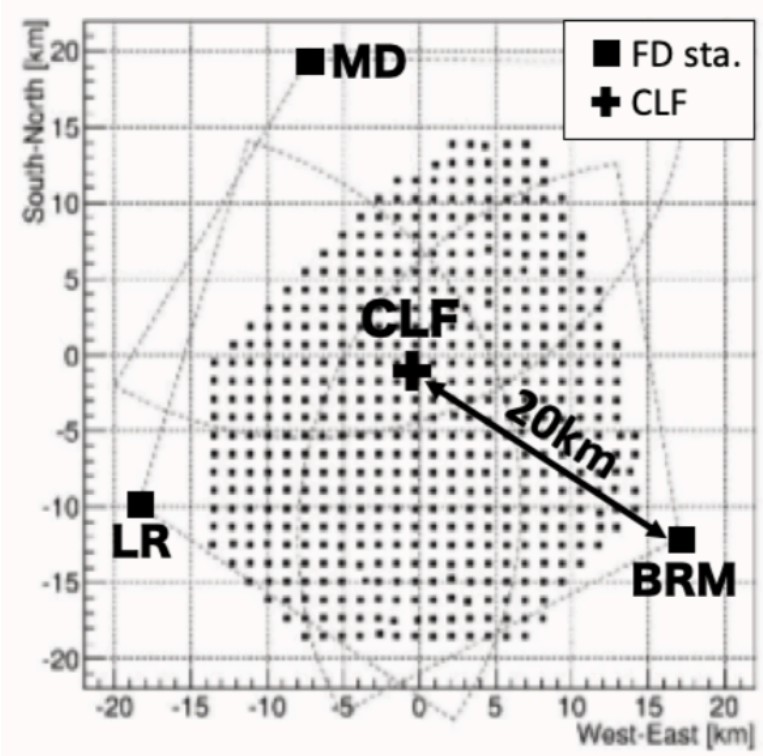

Figure 1: A layout of the TA experiment site. Black small squares are SDs, and three FD stations are shown by $\mathrm{BR}, \mathrm{LR}$, and MD with large square. CLF is located at the center of them.

The Telescope Array experiment keeps observing Ultra High Energy Cosmic Ray(UHECR) from the beginning of 2008 to the present. The TA uses two air shower detection techniques of fluorescence detection technique and particle surface detection technique to observe UHECRs[1][2]. A layout of TA experiment site is shown in Figure 1. The TA consists of 507 surface detectors (SDs) and 3 fluorescence detector(FD) stations. Each FD station called as "Black Rock Mesa"(BR), "Long Ridge"(LR) and "Middle Drum"(MD) have been installed surrounding SD array.

Fluorescence light witch is generated by the air shower. FD observes cosmic rays indirectly by detecting these fluorescence light. Atmospheric monitoring is necessary for observing UHECRs using the air fluorescence technique because the fluorescence light is scattered by the atmosphere in their path of transmission to the FD. Mainly, two types of scattering are considered the atmospherical molecule and the aerosol. Rayleigh scattering that the atmospherical molecule causes can estimate from information of the temperature and the atmospheric pressure obtained from the radiosonde. We use radiosonde data from Elko (Nevada) surrounding the TA site. Effect of Rayleigh scattering to our air shower measurement is relatively small because Rayleigh scattering is determined depending on the atmospherical density. On the other hand, scattering caused by an aerosol has significant effect on energy of cosmic rays because the shape, the amount and the composition of the aerosol are varied by the atmospheric and ground situation. Moreover, they changes time by time.

In the TA experiment, we employ a variety of measurements for atmospheric monitoring, using two laser systems. The first laser system is Mie-LIDAR (LIght Detection And Ranging), which observes the back-scattered light of the laser. Mie-LIDAR is widely used in the ground based aerosol measurement. The Mie-LIDAR system at BR is operated at before the beginning and after 
the end of an FD observation, twice a night. The second laser system is located at the center of three FD stations, and the light scattered by the atmosphere is observed by each fluorescence detector station. This system is called CLF(Central Laser Facility)[3], and UV laser light is vertically burst from the CLF every 30 minutes during FD observations.

\section{Central Laser Facility}
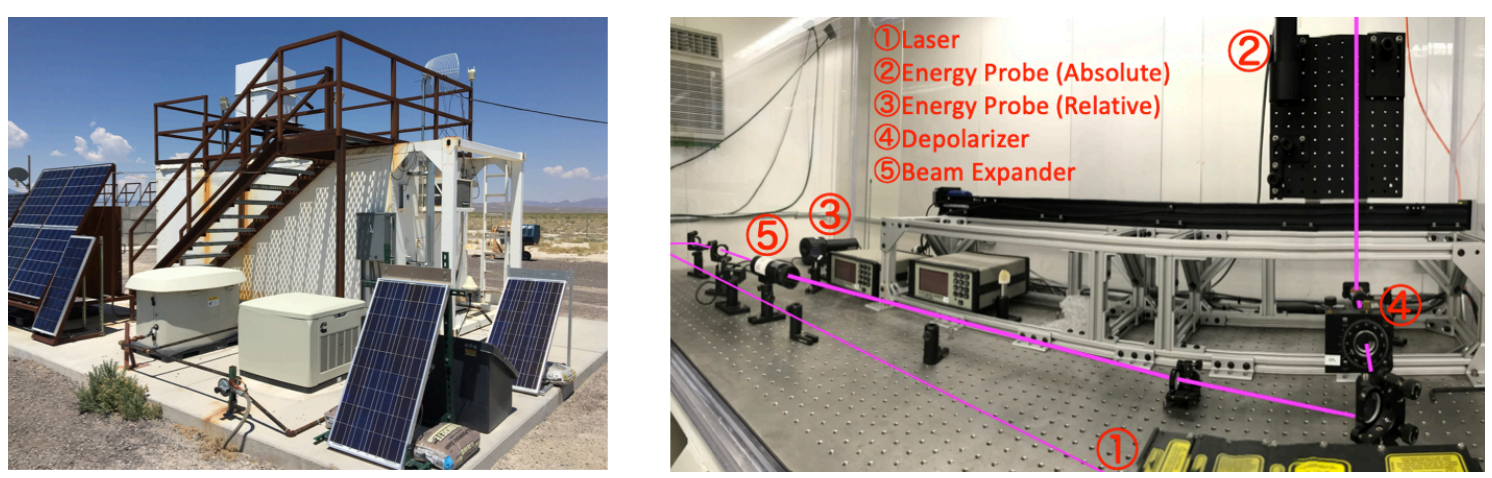

Figure 2: (Left)Outside of CLF container. (Right)Inside of the container and laser optics.

The vertical laser of CLF is burst once every 30 minutes in order to calibrate each FD's detected efficiency including an atmospheric condition. CLF is composed only of the laser shooting part as shown in Figure 2 because the sideway-scattering photon of the laser of CLF is detected by FDs. The laser shot a $355 \mathrm{~nm}$ wavelength with pulse width $6 \sim 8 \mathrm{~ns}$ at $10 \mathrm{~Hz}$ controlled with GPS, the maximum energy of the laser is $10 \mathrm{~mJ}$. Using the CLF system, we can obtain Vertical Aerosol Optical Depth(VAOD) by considering Rayleigh scattering and the detection efficiency of FD. VAOD $\tau_{\mathrm{AS}}$ is a value equal with the integration value of $\alpha$ to $\mathrm{H} \mathrm{km}$ from $0 \mathrm{~km}$ (Equation 2.1). $\alpha$ is extinction coefficient which is the reciprocal of the attenuation length as a function of height $h$.

$$
\tau_{\mathrm{AS}}(\mathrm{H})=\int_{0}^{\mathrm{H}} \alpha_{\mathrm{AS}}(h) d h
$$

VAOD obtained by CLF reflects accurately the total amount of aerosols because the photon passes at a low altitude during the propagation period from the air shower to $\operatorname{FD}$ (see Figure 3). Thus, we can calibration factor of the atmosphere every 30 minutes within the FD observation time. 


\section{VAOD analysis}

\subsection{Method}

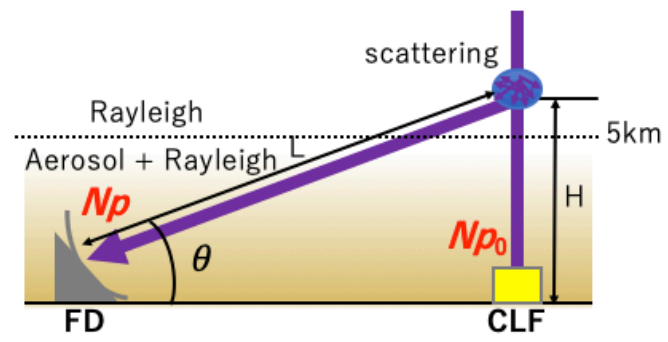

\section{$\mathrm{H}$ : Height of scattering \\ $\mathrm{L}$ : Distance from scattering point to FD \\ $\theta:$ LCLF-FD-Scattering point \\ $N p_{0}:$ Number of photons at the laser injection \\ $N p$ : Amount of light received}

Figure 3: Overview of the laser path in the analysis.

Overview of the laser path in the analysis is shown in Figure [3. The number of photons $N p_{0}$ at laser burst is attenuated by atmospherical molecules and aerosol. The attenuation factor $T(\mathrm{H})$ for photons which vertically propagate in the atmosphere at height $\mathrm{H}$ is given by Equation 3.1

$$
T_{\mathrm{AS}}(\mathrm{H})=\exp \left[-\tau_{\mathrm{AS}}(\mathrm{H})\right]
$$

Suffix "AS" means for "Aerosol", and "Ray" means for "Rayleigh" scattering. After attenuated from the ground to height $\mathrm{H}$ and scattered, the amount of light heading to the FD can be calculated from the scattering coefficient $S$. $S$ is a coefficient including scattering cross-section and angle. The received light amount of FD is shown in Equation B.2.

$$
N p(\mathrm{H})=N p_{0} T_{\text {Ray }}(\mathrm{H}) T_{\mathrm{AS}}(\mathrm{H})\left(S_{\text {Ray }}+S_{\mathrm{AS}}\right) T_{\text {Ray }}(\mathrm{L}) T_{\mathrm{AS}}(\mathrm{L}) .
$$

Assuming the amount of light received $N p^{\prime}$ in the atmosphere without aerosol at the clear night, there is no scattering by aerosol. Therefore, The number of photons $N p_{0}^{\prime}$ at laser burst is scattered by only atmospherical molecules at the clear night. $N p^{\prime}$ at the clear night is shown in Equation 3.3 where it assumed $T_{\mathrm{AS}}(H)=T_{\mathrm{AS}}(L)=1$ and $S_{\mathrm{AS}}=0$.

$$
N p^{\prime}(\mathrm{H})=N p_{0}^{\prime} T_{\text {Ray }}(\mathrm{H}) S_{\text {Ray }} T_{\text {Ray }}(\mathrm{L}) .
$$

With this taken into consideration, we analyze assuming the following two conditions.

- Use scattered at higher than $5 \mathrm{~km}$ height data in measured waveform (atmospherical molecule dominant)

- Assume the atmospheric parameters are constant in horizontal direction

First, Mie-LIDAR by TA experiment has found that aerosols do not exist at a height of $5 \mathrm{~km}$ or more from the ground at the TA site[4]. In this analysis, it is analyzed CLF waveforms of higher than $5 \mathrm{~km}$ of height data because scattering by aerosol is negligible at that height. Therefore, we 
can assume $S_{\mathrm{AS}}=0$. Second, assume that the lateral aerosol distribution for each altitude at the TA experiment site is constant. Thus, attenuation factor $\mathrm{T}(\mathrm{L})$ is calculated by $T(\mathrm{~L})=T(\mathrm{H}) / \sin \theta$. $\theta$ is determined from the light receiving timing recorded by PMTs of FD. Based on these two conditions, VAOD $\tau_{\mathrm{AS}}$ is obtained by comparing $N p$ with $N p^{\prime}$ at the clear night(Equation (3.4).

$$
\frac{N p(\mathrm{H})}{N p^{\prime}(\mathrm{H})}=\frac{N p_{0}}{N p_{0}^{\prime}} \exp \left[-\tau_{\mathrm{AS}}(\mathrm{H}) \frac{\sin \theta+1}{\sin \theta}\right]
$$

\subsection{Data}

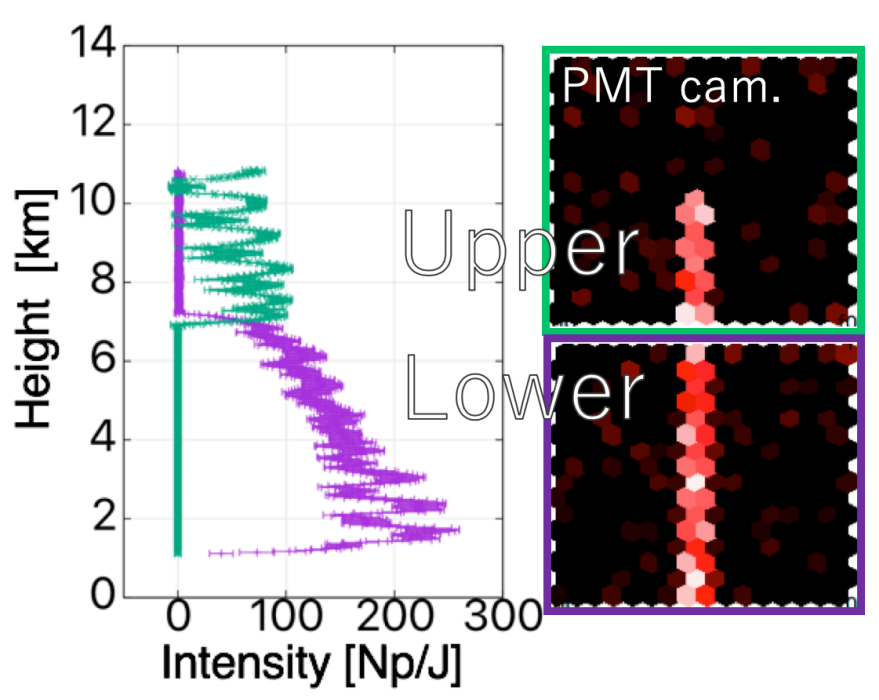

Figure 4: CLF waveform and A map of the amount of light observed by the PMT cameras at that time. The purple plot shows the waveform observed in the lower of the FD and The green plot shows the waveform observed in the Upper of the FD.

The left of Figure 4 shows the time distribution of received light that is normalized by the laser energy. The difference in the output of laser every CLF observation is resolved because the waveform is normalized by the laser energy at then. Therefor, It's possible to compare with waveform by other CLF events. A waveform shows the shape of the unevenness because FD can not be detected the light at the PMT boundary.

The right of Figure 4 shows the map of spatial received light of the field of view of FD. The scattered light at each height are detected by the view of lower and upper FDs.

\subsection{VAOD Result}

We calculated VAOD $\tau_{\mathrm{AS}}(5 \mathrm{~km})$ at the BR station using CLF by 1853 events from January 2012 to September 2016. We divided the period by the maintenance of the equipment etc., and secured three periods that each has one year and analyzed by each period. The distribution of VAOD for 3 years and the transition of VAOD in 1 year is shown in Figure 1 . The median of VAOD 
and $(1 \sigma)$ width of the distribution is $0.043_{-0.015}^{+0.025}$. The VAOD statistic obtained by the Mie-LIDAR system, another atmospheric monitor of TA, is $0.035_{-0.013}^{+0.019}$ [4]. If this analysis is statistically performed under the same conditions (VAOD $\leqq 0.10$ ) as the Mie-LIDAR system, VAOD by our analysis $0.037_{-0.011}^{+0.016}$, that agree with Mie-LIDAR analysis. Figure 6 is shown the seasonal dependence of VAOD. The VAOD is tended to be high in summer (Jun-Sep) and low in winter(Nov-Feb). These signs were also seen in the Mie-LIDAR system. However, the Mie-LIDAR system data provided a little confirmation, because these data are low statistics.

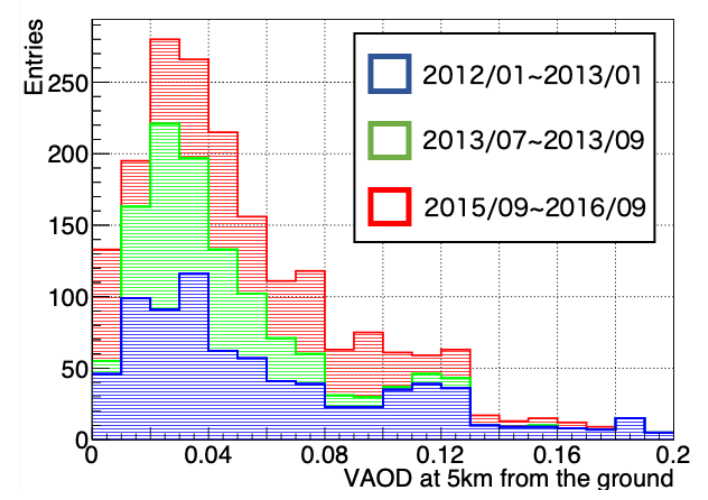

Figure 5: The distribution of VAOD for 3 years.

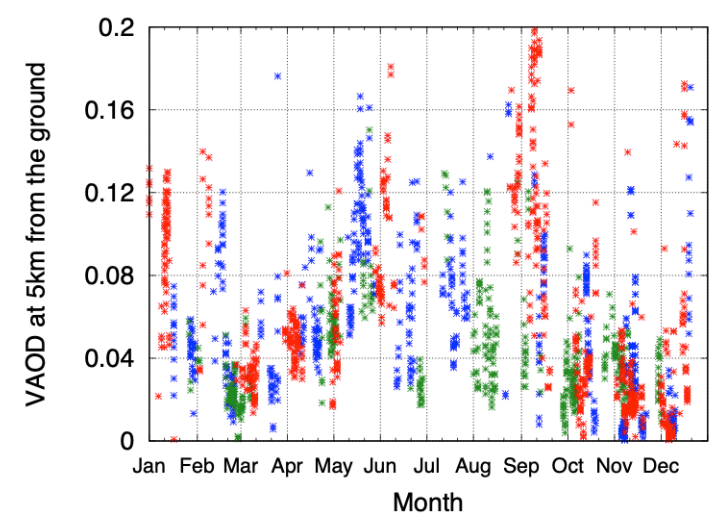

Figure 6: The transition of VAOD in 1 year.

Figure $\square$ shows the distribution of the VAOD in four seasons. Median by the distribution of VAOD in summer is 0.074 and in winter it is 0.029 (Table 1). It is suggested that the VAOD has a seasonal dependence and it has been reported in another experiment. It may be caused by environmental factors such as humidity and temperature.

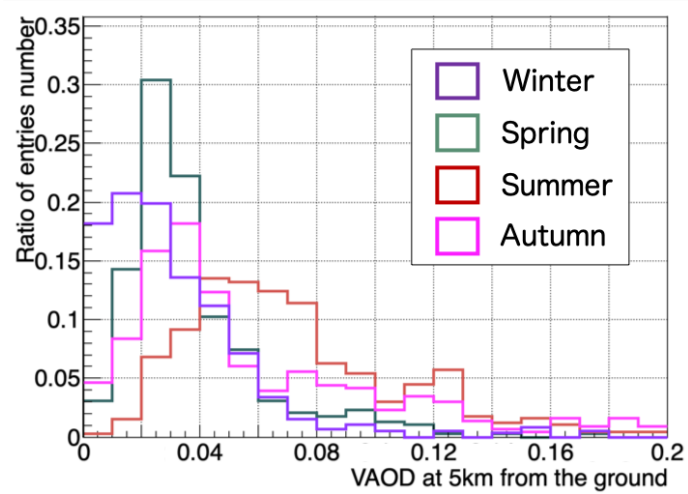

Table 1: The median by the distribution of VAOD in each seasons.

\begin{tabular}{cc}
\hline & Median(VAOD $<0.2)$ \\
\hline Winter & 0.029 \\
Spring & 0.037 \\
Summer & 0.074 \\
Autumn & 0.047 \\
\hline
\end{tabular}

Figure 7: The distribution of VAOD in each seasons.

The VAOD of the BR station compare with the VAOD from another FD site that is the LR station. Figure 8 shows the relationship between VAOD observed at the same time by BR and LR. The VAODs of BR and LR seem to be roughly coincident and distributed around the black line 
showing the same value. Points away from the black line indicate that the atmospheric conditions observed by the two are different, and occasionally the atmospheric transparency has regionality. The distribution of the difference between BR and LR in each observation is shown in Figure 9. The difference between the both is 0.00 on average, and the distribution width of $1 \sigma$ is \pm 0.002 . When VAOD differs by 0.004 that is 2 times of $1 \sigma$ in LR and BR, detected light has difference by $1.5 \%$ reaches to each FD from $5 \mathrm{~km}$ above the CLF.

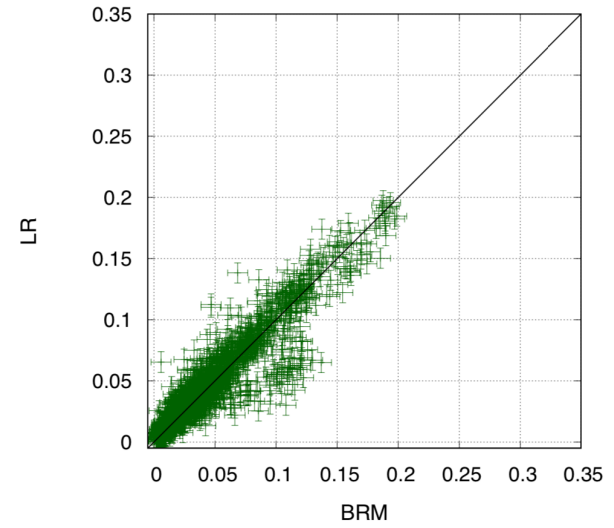

Figure 8: The relationship between VAOD observed at the same time by BR and LR.

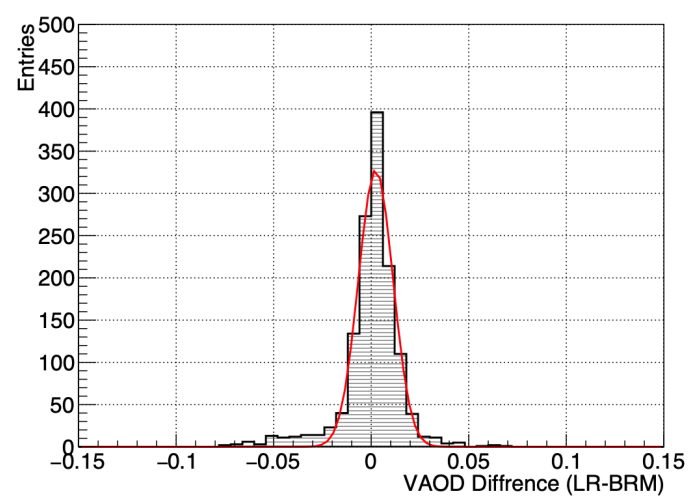

Figure 9: The distribution of the difference between BR and LR.

\section{Conclusions and Future work}

The CLF measures the atmospheric transparency which called Vertical Aerosol Optical Depth. We have found that the median of VAOD at $5 \mathrm{~km}$ from the ground is $0.043_{-0.015}^{+0.025}$ using CLF. The seasonal dependence was confirmed at the transition of VAOD in one year from the VAOD analysis result for 3 years. Near future, we will proceed with the analyses to all the data to get atmospheric transparency and we will modelize seasonally and hourly variation of VAOD. After that, by calibrating the atmospheric transparency by our analysis to UHECRs observed by the FD, we plan to estimate the effect on the reconstruction analysis for the FD.

\section{References}

[1] H.Tokuno et. al., NIM A, 676, 54-65(2012).

[2] T.Abu-Zayyad et. al., NIM A, 689, 87-97(2012).

[3] S. Udo et al., Proceedings of the 30th International Cosmic Ray Conference in Merida, 5(2007) 1021.

[4] T.Takayuki, Nuclear Instruments and Methods in Phisics Research A, 5,653-660(2011). 